PROCEEDINGS OF THE

AMERICAN MATHEMATICAL SOCIETY

Volume 128, Number 11, Pages 3221-3229

S 0002-9939(00)05914-1

Article electronically published on July 6, 2000

\title{
LIPSCHITZ FUNCTIONS WITH MAXIMAL CLARKE SUBDIFFERENTIALS ARE GENERIC
}

\author{
JONATHAN M. BORWEIN AND XIANFU WANG \\ (Communicated by Dale Alspach)
}

\begin{abstract}
We show that on a separable Banach space most Lipschitz functions have maximal Clarke subdifferential mappings. In particular, the generic nonexpansive function has the dual unit ball as its Clarke subdifferential at every point. Diverse corollaries are given.
\end{abstract}

\section{INTRODUCTION AND DEFINITIONS}

We shall be working in a separable Banach space $E$, with norm $\|\cdot\|$, whose topological dual is denoted by $E^{*}$ and whose dual unit ball is denoted by $B^{*}$. For $S \subset E$, a closed convex cone, the positive polar cone is defined by:

$$
S^{+}:=\left\{x^{*} \in E^{*} \mid\left\langle x^{*}, s\right\rangle \geq 0 \text { for every } s \in S\right\} .
$$

Let $A \subset E$ be an open bounded convex set. Throughout $f: A \mapsto R$ denotes a Lipschitz function and is said to be nonexpansive if $|f(x)-f(y)| \leq\|x-y\|$ for all $x, y \in A$ while $f$ is $S$-nondecreasing if $f(y) \geq f(x)$ whenever $y-x \in S$. Note that every function is 0 -nondecreasing. The Clarke derivative [5] at point $x$ in the direction $v$ is given by

$$
f^{0}(x ; v):=\limsup _{\substack{y \rightarrow x \\ t \downarrow 0}} \frac{f(y+t v)-f(y)}{t} .
$$

The Clarke subdifferential $\partial_{c} f$ is given by:

$$
\partial_{c} f(x):=\left\{x^{*} \mid\left\langle x^{*}, v\right\rangle \leq f^{0}(x ; v) \text { for all } v \in E\right\} .
$$

Correspondingly the Michel-Penot derivative [12] at $x$ in the direction $v$ is given by

$$
f^{\diamond}(x ; v):=\sup _{y} \limsup _{t \downarrow 0} \frac{f(x+t y+t v)-f(x+t y)}{t} .
$$

The Michel-Penot subdifferential $\partial_{m p} f$ is then given by:

$$
\partial_{m p} f(x):=\left\{x^{*} \mid\left\langle x^{*}, v\right\rangle \leq f^{\diamond}(x ; v) \text { for all } v \in E\right\},
$$

Received by the editors September 28, 1998.

1991 Mathematics Subject Classification. Primary 49J52; Secondary 26E25, 54E52.

Key words and phrases. Lipschitz function, Clarke subdifferential, separable Banach spaces, Baire category, partial ordering, Banach lattice, approximate subdifferential.

The first author's research was supported by NSERC and the Shrum endowment of Simon Fraser University. 
and is always a convex subset of the Clarke subdifferential but is not usually a closed multifunction. The Dini-Hadamard derivative of $f$ at $x$ in the direction $v$ is

$$
f^{-}(x, v):=\liminf _{t \downarrow 0} \frac{f(x+t v)-f(x)}{t},
$$

and the Dini subdifferential is $\partial_{-} f(x):=\left\{x^{*} \mid\left\langle x^{*}, v\right\rangle \leq f^{-}(x, v)\right.$ for all $\left.v \in E\right\}$. The approximate subdifferential of $f$ at $x[8$ is then defined as:

$$
\partial_{a} f(x):=\bigcap_{\delta>0} w^{*} \operatorname{cl}\left[\partial_{-} f\left(B_{\delta}(x)\right)\right]=\left\{w^{*} \lim _{x_{n} \rightarrow x} x_{n}^{*} \mid x_{n}^{*} \in \partial_{-} f\left(x_{n}\right)\right\},
$$

where the second equality follows from the $w^{*}$-sequential compactness of the dual unit ball, which obtains because $E$ is separable. The central goal of this note is to show that "most" Lipschitz functions have maximal Clarke subdifferential mappings in a sense that we make precise below.

\section{MAIN RESUlT}

Let $C$ be a ${ }^{*}$-compact convex subset of $E^{*}$. By the uniform boundedness theorem, $C$ is norm bounded, say by $K$. Define the support function of $C: \sigma_{C}(s):=$ $\sup \left\{\left\langle s, x^{*}\right\rangle \mid x^{*} \in C\right\}$ for each $s \in E$. $\sigma_{C}$ is sublinear. As $C$ is bounded, $\sigma_{C}$ is finite everywhere with Lipschitz constant $K$ on $E$. Consider

$$
\mathcal{N}_{C}:=\left\{f \mid f: A \mapsto R \text { and } f(x)-f(y) \leq \sigma_{C}(x-y) \text { for all } x, y \in A\right\} .
$$

If $f \in \mathcal{N}_{C}$, then $f(x)-f(y) \leq K\|x-y\|$ for all $x, y \in A$, and so $f$ is Lipschitz. Hence, $\mathcal{N}_{C}$ is a special class of $K$-Lipschitz functions defined on $A$. If $x^{*} \in C$, then

$$
\left\langle x^{*}, x\right\rangle-\left\langle x^{*}, y\right\rangle=\left\langle x^{*}, x-y\right\rangle \leq \sigma_{C}(x-y),
$$

for all $x, y \in A$, thus $x^{*} \in \mathcal{N}_{C}$. From (11), we have $\left\langle x^{*}, x\right\rangle-\sigma_{C}(y) \leq \sigma_{C}(x-y)$, and

$$
\sigma_{C}(x)-\sigma_{C}(y) \leq \sigma_{C}(x-y),
$$

which implies $\sigma_{C} \in \mathcal{N}_{C}$. We continue with three simple lemmas without proof.

Lemma 1. For every $x \in A$, the function $\sigma_{C}(\cdot-x) \in \mathcal{N}_{C}$.

Lemma 2. Assume $f_{\alpha} \in \mathcal{N}_{C}$ for $\alpha \in I$, a finite set. Then $\sup _{\alpha \in I} f_{\alpha} \in \mathcal{N}_{C}$ and $\inf _{\alpha \in I} f_{\alpha} \in \mathcal{N}_{C}$.

The previous lemma remains true for arbitrary index sets when the respective supremum or infimum is finite. On $\mathcal{N}_{C}$ we will use the metric

$$
\rho(f, g):=\sup _{x \in A}|f(x)-g(x)| \text {. }
$$

Lemma 3. For any bounded set $A$, the metric space $\left(\mathcal{N}_{C}, \rho\right)$ is complete.

Thus, in $\left(\mathcal{N}_{C}, \rho\right)$ the Baire category theorem is applicable and every countable intersection of dense open sets is dense: a set containing such a dense $G_{\delta}$ set is called generic or residual and the complement of such a set is meagre. Our central result may now be stated.

Theorem 1. In $\left(\mathcal{N}_{C}, \rho\right)$, the set

$$
\left\{f \in \mathcal{N}_{C} \mid \partial_{c} f \equiv C \text { on } A\right\}
$$

is a residual set. 
Proof. Fix $x \in A$ and $v \in E$. Consider

$$
G_{k}:=\left\{f \in \mathcal{N}_{C} \mid \frac{f(x+t v)-f(x)}{t}-\sigma_{C}(v)>-\frac{1}{k} \text { for some } 0<t<\frac{1}{k}\right\} .
$$

(a) $G_{k}$ is open in $\mathcal{N}_{C}$. Let $f_{0} \in G_{k}$. Then for some $0<t<1 / k$ we have

$$
\frac{f_{0}(x+t v)-f_{0}(x)}{t}-\sigma_{C}(v)>-1 / k \text {. }
$$

Let $\rho\left(f, f_{0}\right)<\varepsilon$ and $f \in \mathcal{N}_{C}$. Consider

$$
\begin{aligned}
& \frac{f(x+t v)-f(x)}{t}-\sigma_{C}(v) \\
& =\frac{(f(x+t v)-f(x))-\left(f_{0}(x+t v)-f_{0}(x)\right)}{t}+\frac{f_{0}(x+t v)-f_{0}(x)}{t}-\sigma_{C}(v) \\
& \geq-\frac{\left|f(x+t v)-f_{0}(x+t v)\right|+\left|f(x)-f_{0}(x)\right|}{t}+\frac{f_{0}(x+t v)-f_{0}(x)}{t}-\sigma_{C}(v) \\
& \geq-\frac{2 \varepsilon}{t}+\frac{f_{0}(x+t v)-f_{0}(x)}{t}-\sigma_{C}(v) .
\end{aligned}
$$

The last expression is greater than $-1 / k$ by equation (2). We may set $\varepsilon$ sufficiently small such that

$$
\frac{-2 \varepsilon}{t}+\frac{f_{0}(x+t v)-f_{0}(x)}{t}-\sigma_{C}(v)>-\frac{1}{k}
$$

Thus, the same $t$ may be used, and so $B\left(f_{0}, \varepsilon\right) \subset G_{k}$.

(b) $G_{k}$ is dense in $\mathcal{N}_{C}$. With $f \in \mathcal{N}_{C}$, for every $\varepsilon>0$ we verify that open ball $B(f, 3 \varepsilon)$ contains a point of $G_{k}$. Define $h: E \mapsto R$ by $h(\tilde{x}):=f(x)-\varepsilon+\sigma_{C}(\tilde{x}-x)$, which is in $\mathcal{N}_{C}$ by Lemma 1 , and set

$$
h_{1}:=\min \{f, h\}, \quad h_{2}:=\max \left\{f-2 \varepsilon, h_{1}\right\} .
$$

Because $f, f-2 \varepsilon, h \in \mathcal{N}_{C}$, Lemma 2 shows $h_{1} \in \mathcal{N}_{C}$, as is $h_{2}$. Since $h_{1} \leq f$ and $f-2 \varepsilon \leq f$, we have $f-2 \varepsilon \leq h_{2} \leq f$. As $f, \sigma_{C}(\cdot-x)$ are continuous at $x$, for $0<\delta<\varepsilon / 2$ sufficiently small we have for $y \in B_{\delta}(x)$,

$$
f(x)-\frac{\varepsilon}{2} \leq f(y) \leq f(x)+\frac{\varepsilon}{2}
$$

and

$$
-\frac{\varepsilon}{2} \leq \sigma_{C}(y-x) \leq \frac{\varepsilon}{2}
$$

Now for $\tilde{x} \in B_{\delta}(x)$ we have

$$
h(\tilde{x}) \leq f(x)-\varepsilon+\varepsilon / 2 \leq f(x)-\frac{\varepsilon}{2} \leq f(\tilde{x}),
$$

and so $h_{1}=\min \{f, h\}=h$ on $B_{\delta}(x)$. On the other hand, on $B_{\delta}(x)$ by equations (4) and (3) we have

$$
h_{1}(\tilde{x})=h(\tilde{x}) \geq f(x)-\varepsilon-\varepsilon / 2 \geq f(x)-\frac{3 \varepsilon}{2}
$$

and

$$
f(\tilde{x})-2 \varepsilon \leq f(x)-\frac{3 \varepsilon}{2},
$$


and so $h_{2}=h_{1}=h$ on $B_{\delta}(x)$. Choosing $0<t<1 / k$ sufficiently small such that $x+t v \in B_{\delta}(x)$, we have

$$
\begin{aligned}
\frac{h_{2}(x+t v)-h_{2}(x)}{t} & =\frac{h(x+t v)-h(x)}{t} \\
& =\frac{f(x)-\varepsilon+\sigma_{C}(t v)-(f(x)-\varepsilon)}{t}=\sigma_{C}(v),
\end{aligned}
$$

which shows $h_{2} \in G_{k}$ while $h_{2}$ is arbitrarily close to $f$.

(c) Since $G_{k}$ is open and dense in $\mathcal{N}_{C}$,

$$
G_{x, v}:=\bigcap_{k=1}^{\infty} G_{k}
$$

is a dense $G_{\delta}$ set in $\mathcal{N}_{C}$. If $f \in G_{x, v}$, then for every $k$ we can find $0<t_{k}<1 / k$ such that

$$
\frac{f\left(x+t_{k} v\right)-f(x)}{t_{k}}-\sigma_{C}(v)>-\frac{1}{k},
$$

and taking the limit we derive

$$
f^{0}(x, v) \geq f^{\diamond}(x, v) \geq \limsup _{t \downarrow 0} \frac{f(x+t v)-f(x)}{t} \geq \sigma_{C}(v) .
$$

(d) Now let $\left\{v_{k}\right\}$ be a norm dense countable set in $E$. For each $v_{k}$, the set $G_{x, v_{k}}$ is a dense $G_{\delta}$ set in $\mathcal{N}_{C}$. Hence,

$$
G_{x}:=\bigcap_{k=1}^{\infty} G_{x, v_{k}}
$$

is also a dense $G_{\delta}$ set in $\mathcal{N}_{C}$.

Given $f \in G_{x}$ we note that for each $k$ we have

$$
f^{0}\left(x, v_{k}\right) \geq f^{\diamond}\left(x, v_{k}\right) \geq \sigma_{C}\left(v_{k}\right) .
$$

Because $f^{0}(x, \cdot), f^{\diamond}(x, \cdot)$ and $\sigma_{C}(\cdot)$ are Lipschitz, we deduce

$$
f^{0}(x, v) \geq f^{\diamond}(x, v) \geq \sigma_{C}(v),
$$

for every $v \in E$.

(e) Finally let $\left\{x_{k}\right\}$ be a norm dense countable set in $A$. Since each $G_{x_{k}}$ is a dense $G_{\delta}$ set in $\mathcal{N}_{C}$, the set

$$
G:=\bigcap_{k=1}^{\infty} G_{x_{k}}
$$

is also a dense $G_{\delta}$ set in $\mathcal{N}_{C}$. For $f \in G$, and each positive integer $k$ we have $f^{0}\left(x_{k}, v\right) \geq \sigma_{C}(v)$ for all $v \in E$. Since $f^{0}(x, v)$ is upper semicontinuous in $x$, we obtain

$$
f^{0}(x, v) \geq \sigma_{C}(v)
$$

for every $x \in A$ and $v \in E$.

Since $f \in \mathcal{N}_{C}$, for every $v \in E$, we have $f^{0}(x, v)=$

$$
\limsup _{\substack{y \rightarrow x \\ t \downarrow 0}} \frac{f(y+t v)-f(y)}{t} \leq \limsup _{\substack{y \rightarrow x \\ t \downarrow 0}} \frac{\sigma_{C}(y+t v-y)}{t}=\limsup _{t \downarrow 0} \frac{\sigma_{C}(t v)}{t}=\sigma_{C}(v) .
$$


Then for $f \in G$, we have $f^{0}(x, v)=\sigma_{C}(v)$ for every $x \in A$ and $v \in E$. Dually, $\partial_{c} f \equiv C$ on $A$.

Unbounded domains are only slightly more difficult to handle. In particular, let us now consider:

$$
\mathcal{X}_{C}:=\left\{f: E \mapsto R \mid f(x)-f(y) \leq \sigma_{C}(x-y) \text { for } x, y \in E\right\} .
$$

Define the metric of uniform convergence on bounded sets, $\tilde{\rho}$, on $\mathcal{X}_{C}$ by

$$
\tilde{\rho}(f, g):=\sum_{n=1}^{\infty} \frac{1}{2^{n}} \frac{\rho_{n}(f, g)}{1+\rho_{n}(f, g)} \quad \text { where } \rho_{n}(f, g):=\sup _{x \in n B}|f(x)-g(x)| .
$$

For the metric $\tilde{\rho}$ (or many variants, for example $\tilde{\rho}(f, g):=\sum_{n=1}^{\infty} 1 / 2^{n} \rho_{n}(f, g)$ ), $f \rightarrow g$ if and only if $f \rightarrow g$ on $n B$ in the metric $\rho_{n}$ for every $n$. In an entirely standard fashion, we may verify that $\left(\mathcal{X}_{C}, \tilde{\rho}\right)$ is complete. For fixed $x, v$, with $f_{0}, t$ as in equation (2) there exists some integer $N>0$ such that $\|x\| \leq N,\|x+t v\| \leq N$. By definition of $\tilde{\rho}$,

$$
\frac{1}{2^{N}} \frac{\rho_{N}\left(f, f_{0}\right)}{1+\rho_{N}\left(f, f_{0}\right)} \leq \tilde{\rho}\left(f, f_{0}\right) .
$$

For $\tilde{\rho}\left(f, f_{0}\right)<\varepsilon$, we have $\rho_{N}\left(f, f_{0}\right) \leq\left(2^{N} \varepsilon\right) /\left(1-2^{N} \varepsilon\right)$. Thus for small $\varepsilon$, the same argument in (a) applies to guarantee $G_{k}$ being open. The arguments in (b), (c), (d), (e) still apply. Hence:

Corollary 1. In $\left(\mathcal{X}_{C}, \tilde{\rho}\right)$, the set

$$
\left\{f \in \mathcal{X}_{C} \mid \partial_{c} f \equiv C \text { on } E\right\}
$$

is residual.

One should compare Corollary 1$]$ with the following result due to Jouini [1]:

Proposition 1. Let $K$ be a nonempty convex compact subset of $R^{n}$. Then there exists a Lipschitz function $F_{K}: R^{n} \mapsto R$ such that, for all $x \in R^{n}, K=\partial_{c} F_{K}(x)$.

We have generalized Jouini's result in three ways: (i) from $R^{n}$ to an arbitrary separable Banach space, (ii) Lipschitz functions with maximal subdifferential are generic, (iii) our technique is much simpler and the proof less ambiguous.

When $C:=\left\{x^{*}\right\}$, a singleton, for every $f \in \mathcal{N}_{C}$ we have $f(x)-f(y)=\left\langle x^{*}, x-y\right\rangle$ for $x, y \in A$. Thus $\mathcal{N}_{C}$ consists of all affine functionals of the form $\beta+\left\langle x^{*}, \cdot\right\rangle$. Every $f \in \mathcal{N}_{C}$ then has gradient $x^{*}$. Thus, it is only interesting to consider $C$ being nonsingleton in the sequel. We next record a result for the Michel-Penot subdifferential.

Corollary 2. For every countable (dense) set $D \subset A$, the set

$$
\mathcal{N}_{C}(D):=\left\{f \in \mathcal{N}_{C} \mid \partial_{m p} f(x)=C \text { for each } x \in D\right\}
$$

is residual in $\mathcal{N}_{C}$.

Proof. From step (d) of Theorem 1 for $x \in A, G_{x}$ is a dense $G_{\delta}$ set in $\mathcal{N}_{C}$. If $f \in G_{x}$, we have

$$
f^{\diamond}(x, v) \geq \sigma_{C}(v) \quad \text { for every } v \in E,
$$

and dually $\partial_{m p} f(x) \supset C$. As $f \in \mathcal{N}_{C}$, we have

$$
f^{\diamond}(x, v) \leq f^{0}(x, v) \leq \sigma_{C}(v) \text { for every } v \in E
$$


then $\partial_{m p} f(x) \subset C$. Thus, $\partial_{m p} f(x)=C$. For a countable set $D$, the set $G:=$ $\bigcap_{x \in D} G_{x}$ is the required residual set in $\mathcal{N}_{C}$.

The Michel-Penot subdifferential agrees with the Gâteaux derivative whenever the latter exists, and the separable Banach space version of Rademacher's theorem [4] ensures members of $\mathcal{N}_{C}$ are Gâteaux differentiable except on a Haar-null set. Thus, no member of $\mathcal{N}_{S}$ can have $\partial_{m p} f(x)=C$ except on a null set.

Noting that

$$
\mathcal{N}_{B^{*}}:=\{f \mid f: A \mapsto R \text { is nonexpansive with respect to }\|\cdot\|\},
$$

we obtain:

Corollary 3. In the space of nonexpansive functions, $\left(\mathcal{N}_{B^{*}}, \rho\right)$, the set

$$
\left\{f \in \mathcal{N}_{B^{*}} \mid \partial_{c} f \equiv B^{*} \text { on } A\right\}
$$

is a residual set.

When $E:=R^{1}$, this provides a generic version of Rockafellar's original example [13. More generally, it provides generic examples of some of the constructions in [2].

Remarks 1. (i) In $\left(\mathcal{N}_{B^{*}}, \rho\right)$, uncountably many nonexpansive functions not differing by constants have maximal Clarke's subdifferentials. To see this, fix $x_{0} \in A$ and define $P(f):=f-f\left(x_{0}\right)$. Then $\left(P\left(\mathcal{N}_{B^{*}}\right), \rho\right)$ is a complete metric space without isolated points, and $\left\{f \in \mathcal{N}_{B^{*}} \mid \partial_{c} f \equiv B^{*}\right.$ on $\left.A, f\left(x_{0}\right)=0\right\}$ is residual in $\left(P\left(\mathcal{N}_{B^{*}}\right), \rho\right)$. In a complete metric space without isolated points, each residual set is uncountable.

(ii) Every $C^{1}$ "tube" is a Clarke-subdifferential map. Indeed, by Corollary 3 we may take $f: A \mapsto R$ with $\partial_{c} f \equiv B^{*}$. Let $g: A \mapsto R$ be locally Lipschitz and strictly Gâteaux differentiable on $A$. Then by Proposition 2.3.3 in [5] we have $\partial_{c}(f+g)(x)=\nabla g(x)+B^{*}$ for all $x \in A$.

Suppose $f \in \mathcal{N}_{C}$ is $S$-nondecreasing. For $v \in S, x \in A$ we have

$$
-f^{0}(x,-v)=-\limsup _{\substack{y \rightarrow x \\ t \downarrow 0}} \frac{f(y+t(-v))-f(y)}{t}=\liminf _{\substack{y \rightarrow x \\ t \downarrow 0}} \frac{f(y)-f(y-t v)}{t} \geq 0 .
$$

If $x^{*} \in \partial_{c} f(x)$, then $\left\langle x^{*}, v\right\rangle \geq-f^{0}(x,-v) \geq 0$. Thus $x^{*} \in S^{+}$. Together with equation (5), we obtain $\partial_{c} f \subset C \cap S^{+}$. By Lebourg's mean value theorem [5] for every $x, y \in A$ there exists $u \in(x, y)$ such that

$$
f(x)-f(y)=\left\langle x^{*}, x-y\right\rangle \leq \sigma_{C \cap S^{+}}(x-y),
$$

where $x^{*} \in \partial_{c} f(u)$. It follows that $f \in \mathcal{N}_{C \cap S^{+}}$. Conversely, if $f \in \mathcal{N}_{C \cap S^{+}} \subset \mathcal{N}_{C}$, for every $x \in A$ and $s \in S$ with $x+s \in A$ we have

$$
f(x)-f(x+s) \leq \sigma_{C \cap S^{+}}(-s) \leq 0,
$$

which implies $f$ is $S$-nondecreasing. In other words,

$$
\mathcal{N}_{C \cap S^{+}}=\left\{f \in \mathcal{N}_{C} \mid f \text { is } S \text {-nondecreasing }\right\} .
$$

Corollary 4. In $\left(\mathcal{N}_{C \cap S^{+}}, \rho\right)$, the set

$$
\left\{f \in \mathcal{N}_{C \cap S^{+}} \mid \partial_{c} f \equiv C \cap S^{+} \text {on } A\right\}
$$

is a residual set. 
Recall as in [9, page 369] that a partially ordered set is a lattice if every twopoint set $\{x, y\}$ has a supremum and an infimum, denoted by $x \vee y$ and $x \wedge y$ respectively. A partially ordered linear space in which this condition holds is called a linear lattice. Define $|x|:=x \vee(-x), x^{+}=x \vee 0$. A normed lattice is a linear lattice with a norm such that $|x| \leq|y|$ implies $\|x\| \leq\|y\|$. A normed lattice that is also complete is called a Banach lattice. Classical separable Banach lattices are: $c_{0}$ with the supremum norm and $l^{p}$ for $1 \leq p<+\infty$ with $p$-norm for the ordering $x \leq y$ defined by $x(n) \leq y(n)$ for all $n, L_{p}[0,1]$ for $1 \leq p<+\infty$ with the $p$-norm and the ordering $f \leq g$ defined by $f(p) \leq g(p)$ almost everywhere on $[0,1]$, and $C[0,1]$ with the supremum norm and pointwise order. By equation (6),

$\mathcal{N}_{B^{*} \cap S^{+}}:=\{f \mid f: A \mapsto R$ is nonexpansive with respect to $\|\cdot\|$ and $S$-nondecreasing\}.

Corollary 5. In a separable Banach lattice $E$ with $S:=\{x \in E \mid x \geq 0\}$, the set

$$
\left\{f \in \mathcal{N}_{B^{*} \cap S^{+}} \mid \partial_{c} f \equiv\left(B^{*}\right)^{+} \text {on } A\right\}
$$

is a residual set in $\left(\mathcal{N}_{B^{*} \cap S^{+}}, \rho\right)$, the space of nondecreasing and nonexpansive functions.

Example 1. Cornet 11 formalized a nonsmooth marginal price rule in mathematical economics by establishing that given a closed production set $Y$ the price $p \in N_{Y}(y)$ for all $y$ in the boundary of $Y$. Here $N_{Y}(y)$ is the Clarke normal cone of $Y$ at $y$. Take $f: E \mapsto R$ with $\partial_{c} f(x) \equiv C$. The epigraph of $f$ as always is defined as:

$$
\text { epi } f:=\{(x, r) \in E \times R: f(x) \leq r\} .
$$

The Clarke tangent cone and normal cone to the epigraph of $f$ at $(x, f(x))$ are then constant set-valued maps:

$$
T_{e p i f}(x, f(x))=\left\{(v, \beta) \mid \sigma_{C}(v) \leq \beta\right\} \text { and } N_{e p i f}(x, f(x))=\bigcup_{\lambda \geq 0} \lambda[C,-1] .
$$

For every $(x, r) \in$ epi $f$ and $(v, \beta) \in T_{\text {epi } f}(x, f(x))$ we have

$$
f(x+v) \leq f(x)+\sigma_{C}(v) \leq r+\beta,
$$

thus epif $+T_{\text {epi } f} \subset$ epi $f$.

In $R^{n}$, if we take $0 \in C \subset R^{n-1}$ with $n$ extreme points $v_{1}, \ldots, v_{n}$ such that

$$
\left\langle\left(v_{i},-1\right),\left(v_{j},-1\right)\right\rangle=0 \text { for } i \neq j,
$$

then $N_{\text {epi } f}(x, f(x))$ is the closed convex cone generated by $\left(v_{1},-1\right), \ldots,\left(v_{n},-1\right)$ which is linearly isometric to $R_{n}^{+}$. Thus $T_{e p i f}(x, f(x))$ is linearly isometric to $R_{n}^{-}$. Then epi $f$ is isometric to a closed set $Y \subset R^{n}$ such that $N_{Y}(y)=R_{n}^{+}$for $y$ in the boundary of $Y$ and $Y-R_{n}^{+} \subset Y$ ("free disposal"). Thus the marginal rule generically imposes no restriction on the price vector.

Let us define the oscillation of $f$ at $x$ by

$$
\Omega_{H}^{f}(x):=\left\{w^{*} \lim _{x_{n} \rightarrow x} \nabla f\left(x_{n}\right) \mid x_{n} \notin H\right\},
$$

where $H$ is any Haar-null set containing the set of points at which $f$ fails to be Gâteaux differentiable. Since $E$ is separable, the dual unit ball $B^{*}$ is metrizable 
in the $\mathrm{w}^{*}$ topology. The sequence $\left(\nabla f\left(x_{n}\right)\right)$ thus has $\mathrm{w}^{*}$ convergent subsequences and hence $\Omega_{H}^{f}(x) \neq \emptyset$. In [14] Thibault showed that $\Omega_{H}^{f}(x)$ is $\mathrm{w}^{*}$ compact and

$$
\partial_{c} f(x)=w^{*} \operatorname{cl}-\operatorname{conv}\left[\Omega_{H}^{f}(x)\right],
$$

so $w^{*} \operatorname{cl}-\operatorname{conv}\left[\partial_{a} f(x)\right]=\partial_{c} f(x)$. Denote the norm on $E^{*}$ by $\|\cdot\|_{*}$ and denote the unit sphere by $U^{*}:=\left\{x^{*} \in B^{*} \mid\left\|x^{*}\right\|_{*}=1\right\}$.

Lemma 4. Assume $E$ is an infinite dimensional Banach space. Then the dual sphere is $w^{*}$ sequentially dense in the dual ball:

$$
B^{*}=\left\{x^{*} \mid \text { there exists } x_{n}^{*} \stackrel{w^{*}}{\rightarrow} x^{*} \text { with }\left\|x_{n}^{*}\right\|_{*}=1\right\} .
$$

Proof. If $E$ is separable, this is immediate from the metrizability of the dual ball. We give the proof in full generality. Assume $\left\|x^{*}\right\|_{*}<1$. By the JosefsonNissenszweig theorem [6] there is a sequence $y_{n}^{*} \stackrel{w^{*}}{\rightarrow} 0$ with $\left\|y_{n}^{*}\right\|_{*}=1$. Define $\phi(t):=\left\|t y_{n}^{*}+x^{*}\right\|_{*}$. As

$$
\phi\left(1+\left\|x^{*}\right\|_{*}\right) \geq\left(1+\left\|x^{*}\right\|_{*}\right)\left\|y_{n}^{*}\right\|_{*}-\left\|x^{*}\right\|_{*}=1
$$

and $\phi(0)=\left\|x^{*}\right\|_{*}<1$, we may choose $t_{n} \in\left[0,1+\left\|x^{*}\right\|_{*}\right]$ such that $\left\|t_{n} y_{n}^{*}+x^{*}\right\|_{*}=1$. Define $x_{n}^{*}:=t_{n} y_{n}^{*}+x^{*}$. As $y_{n}^{*} \stackrel{w^{*}}{\rightarrow} 0$ and $\left\{t_{n}\right\}$ is bounded, we obtain $x_{n}^{*} \stackrel{w^{*}}{\rightarrow} x^{*}$.

Lemma 5. Assume $E$ is a Banach space with a Gâteaux differentiable norm $\|\cdot\|$. Then the set of extreme points of $B^{*}$, denoted by ext $\left(B^{*}\right)$, is norm dense in $U^{*}$.

Proof. Take $\left\|x^{*}\right\|_{*}=1$. For every $1>\varepsilon>0$, by the Bishop-Phelps theorem, there exists $y^{*} \in E^{*}$ with $\left\|y^{*}-x^{*}\right\|_{*}<\varepsilon / 2$ such that $y^{*}(y)=\left\|y^{*}\right\|_{*}$ for some $\|y\|=1$. Then $1-\varepsilon / 2<\left\|y^{*}\right\|_{*}<1+\varepsilon / 2$ and

$$
\left\|\frac{y^{*}}{\left\|y^{*}\right\|_{*}}-x^{*}\right\|_{*} \leq\left\|\frac{y^{*}}{\left\|y^{*}\right\|_{*}}-y^{*}\right\|_{*}+\left\|y^{*}-x^{*}\right\|_{*}<\varepsilon
$$

We show that $y^{*} /\left\|y^{*}\right\|_{*}$ is an extreme point of $U^{*}$. Indeed, if $y^{*} /\left\|y^{*}\right\|_{*}=\lambda z_{1}^{*}+$ $(1-\lambda) z_{2}^{*}$ for some $0<\lambda<1$ with $\left\|z_{1}^{*}\right\|_{*}=1,\left\|z_{2}^{*}\right\|_{*}=1$, then

$$
1=\frac{y^{*}}{\left\|y^{*}\right\|_{*}}(y)=\lambda z_{1}^{*}(y)+(1-\lambda) z_{2}^{*}(y)
$$

implies $z_{1}^{*}(y)=1=z_{2}^{*}(y)$. We thus have $z_{1}^{*}, z_{2}^{*} \in \partial\|\cdot\|(y)$. The Gâteaux differentiability of $\|\cdot\|$ at $y$ implies $z_{1}^{*}=z_{2}^{*}$.

Theorem 2. Assume $E$ is an infinite dimensional separable Banach space with Gâteaux differentiable norm. In $\left(\mathcal{N}_{B^{*}}, \rho\right)$,

(i) the set $\left\{f \in \mathcal{N}_{B^{*}} \mid \Omega_{H}^{f}(x) \equiv B^{*}\right.$ on $\left.A\right\}$ is a residual set; and so

(ii) the set $\left\{f \in \mathcal{N}_{B^{*}} \mid \partial_{a} f(x) \equiv B^{*}\right.$ on $\left.A\right\}$ is a residual set.

Proof. Assume $f$ has $\partial_{c} f \equiv B^{*}$. By equation (7), $w^{*} \operatorname{cl}-\operatorname{conv}\left[\Omega_{H}^{f}(x)\right]=B^{*}$. From the Krein-Milman converse theorem [7, page 74], we have

$$
\operatorname{ext}\left(B^{*}\right) \subset w^{*}-\operatorname{cl}\left[\Omega_{H}^{f}(x)\right]=\Omega_{H}^{f}(x) .
$$

Lemma 5 shows $\operatorname{ext}\left(B^{*}\right)$ is norm dense in the sphere $U^{*}$, and so

$$
U^{*} \subset w^{*}-\operatorname{cl}\left[\operatorname{ext}\left(B^{*}\right)\right] \subset \Omega_{H}^{f}(x),
$$

by the $\mathrm{w}^{*}-$ closedness of $\Omega_{H}^{f}(x)$. From Lemma 4

$$
B^{*}=w^{*}-\operatorname{cl}\left[U^{*}\right] \subset \Omega_{H}^{f}(x) \subset B^{*} .
$$


(i) follows by applying Corollary 3. Then (ii) follows from $\Omega_{H}^{f}(x) \subset \partial_{a} f(x) \subset$ $\partial_{c} f(x)$ and (i).

Theorem 2 holds isometrically in separable Hilbert space and the classical $\mathcal{L}_{p}$ spaces $(1<p<\infty)$ and isomorphically in every infinite dimensional separable space since each separable Banach space has an equivalent Gâteaux differentiable norm (with its dual norm being strictly convex). Theorem 2 (ii) also holds in $R$ by [3]. Sadly we do not know whether this is true in $R^{n}$ with $2 \leq n<+\infty$.

Finally we note that in [1] a careful study was made of those Lipschitz functions whose Clarke's subdifferentials are minimal with respect to set inclusion, viewed as norm to $\mathrm{w}^{*}$ upper semicontinuous multifunctions with nonempty convex compact images. These functions capture most of the concrete classes of Lipschitz functions occurring in practice - convex functions, smooth functions, distance functions in appropriately smooth norms, etc. Nonetheless, from Corollary 3 we obtain:

Corollary 6. On a separable Banach space, the set of nonexpansive functions with minimal Clarke subdifferentials is first category in $\left(\mathcal{N}_{B^{*}}, \rho\right)$.

\section{REFERENCES}

[1] J.M. Borwein, W.B. Moors, Essentially smooth Lipschitz functions, J. Funct. Anal. 149 (1997), 305-351. MR 98i:58028

[2] J.M. Borwein, W.B. Moors and Xianfu Wang, Lipschitz functions with prescribed derivatives and subderivatives, Nonl. Anal. Theor. Meth. Appl. 29 (1997), 53-64. MR 98j:49019

[3] J.M. Borwein, S. Fitzpatrick, Characterization of Clarke subgradients among one-dimensional multifunctions, in Proc. of the Optimization Miniconference II, edited by B. M. Glover and V. Jeyakumar, (1995), 61-73.

[4] J.P.R. Christensen, Measure theoretic zero sets in infinite dimensional spaces and applications to differentiability of Lipschitz mappings, II, Coll. Anal. Funct. Bordeaux (1973), 29-39. MR 50:14215

[5] F.H. Clarke, Optimization and Nonsmooth Analysis, Wiley Interscience, New York, 1983. MR 85m:49002

[6] J. Diestel, Sequences and Series in Banach Spaces, Springer-Verlag, New York, 1984. MR 85i:46020

[7] R.B. Holmes, Geometric Functional Analysis and its Applications, Springer-Verlag, New York, 1975. MR 53:14085

[8] A.D. Ioffe, Approximate subdifferentials and applications 3: the metric theory, Mathematika, 36 (1989), 1-38. MR 90g:49012

[9] G.J.O. Jameson, Topology and Normed Spaces, Chapman and Hall, 1974. MR 57:3828

[10] E. Jouini, A remark on Clarke's normal cone and the marginal cost pricing rule, J. Math. Econom. 18 (1989), 95-101. MR 90i:90026

[11] E. Jouini, Functions with constant generalized gradients, J. Math. Anal. Appl. 148 (1990), 121-130. MR 91c:90100

[12] Ph. Michel, J.P. Penot, Calcul sous-différential pour des fonctions Lipschitziennes et nonLipschiziennes, C. R. Acad. Sci. Paris, Ser. I Math., 298 (1984), 269-272. MR 85i:49027

[13] R.T. Rockafellar, The Theory of Subgradients and Its Applications to Problems of Optimization: Convex and Nonconvex Functions, Helderman Verlag, Berlin, 1981. MR 83b:90126

[14] L. Thibault, On generalized differentials and subdifferentials of Lipschitz vector-valued functions, Nonl. Anal. Theor. Meth. Appl. 6 (1982), 1037-1053. MR 85e:58020

Centre for Experimental and Constructive Mathematics, Department of Mathematics and Statistics, Simon Fraser University, Burnaby, British Columbia, Canada V5A $1 \mathrm{~S} 6$

E-mail address: jborwein@cecm.sfu.ca

E-mail address: xwang@cecm.sfu.ca 